\title{
CLARIFICAÇÃO DO CALDO DE CANA-DE-AÇÚCAR UTILIZANDO A REAÇÃO DE PEROXIDAÇÃO COM INCIDÊNCIA DE LUZ ULTRAVIOLETA
}

\section{CLARIFICATION OF SUGARCANE JUICE USING PEROXIDATION REACTION WITH ULTRAVIOLET LIGHT INCIDENCE}

\author{
Á. O. S. DANTAS ${ }^{1, *}$, L. C. S. CAVAlCANTE ${ }^{2}$, G. E. SANTOS $^{1}$, C. L. P. S. ZANTA ${ }^{3}$, S. H. V. \\ CARVALHO ${ }^{2}$
}

${ }^{1}$ Universidade Federal de Uberlândia, Faculdade de Engenharia Química, Uberlândia, Minas Gerais, Brasil

${ }^{2}$ Universidade Federal de Alagoas, Centro de Tecnologia, Maceió, Alagoas, Brasil

${ }^{3}$ Universidade Federal de Alagoas, Instituto de Química e Biotecnologia, Maceió, Alagoas, Brasil

${ }^{*}$ Corresponding author. Federal University of Uberlândia, Department of Chemical Engineering, Uberlândia, Minas Gerais, Brazil, Phone: +55 $343239-4249$ e-mail address: adilas.dantas@gmail.com (Á. O. S. Dantas).

\begin{tabular}{l} 
A R T I C L E I N F O \\
\hline Article history: \\
Received 2020-05-19 \\
Accepted 2020-09-04 \\
Available online 2020-09-04 \\
pa la v r a s - ch a ve \\
Clarificação \\
Peróxido de Hidrogênio \\
Luz Ultravioleta \\
Cana-de-açúcar \\
ke yw or $d s$ \\
Clarification \\
Hydrogen Peroxide \\
Ultraviolet Light \\
Sugarcane \\
\end{tabular}

\begin{abstract}
A B S T R A C T
Currently, sulphitation process is used in clarification and posterior commercial sugar production, which has created controversial relations regarding the impacts on consumers' health. Alternative methods have been widely studied to replace sulfur-based compounds. Hydrogen peroxide reaction as a clarifying agent under ultraviolet light is one of the promising and effective advanced oxidative processes (AOPS). This paper evaluated this process with sugarcane juice aiming to produce a higher value-added sugar, using two concentrations $(0.5$ and $1.0 \mathrm{~mol} / \mathrm{L})$ of $\mathrm{H}_{2} \mathrm{O}_{2}$ and incidence or absence of ultraviolet light. According to the results, clarification was up to $55 \%$ when using $0.5 \mathrm{~mol} / \mathrm{L}$ hydrogen peroxide concentration under the effect of UV light, thus conferring that the alternative method is viable.
\end{abstract}

R E S UM O

Atualmente, para clarificação e fabricação do açúcar comercial, utiliza-se o processo de sulfitação, que tem gerado controvérsias com relação ao impacto na saúde dos consumidores. Métodos alternativos são estudados no intuído de substituir os compostos à base de enxofre. A reação do peróxido de hidrogênio sob a presença de luz ultravioleta é um dos processos oxidativos avançados promissores, e comprovadamente eficaz. O presente trabalho avaliou este processo para o caldo de cana-de-açúcar com o objetivo de produzir um açúcar de maior valor agregado, utilizando duas concentrações $\left(0,5\right.$ e 1,0 mol/L) de $\mathrm{H}_{2} \mathrm{O}_{2}$ e considerando os efeitos da luz ultra-violeta. De acordo com os resultados, pôde-se obter uma clarificação de até $55 \%$, quando se utiliza concentrações de 0,5 mol/L de peróxido de hidrogênio sob o efeito da luz UV, mostrando assim, que há viabilidade nesse método alternativo. 


\section{INTRODUÇ̃̃̃O}

O Brasil é o maior produtor de açúcar mundial, sendo a cana-de-açúcar a principal cultura responsável por esse commoditie (Ogando et al., 2019). As indústrias sucroalcooleiras processam uma mistura de variedades de canade-açúcar, na tentativa de enquadrar a cor do açúcar aos parâmetros de qualidade do mercado consumidor.

A cor é um dos mais importantes parâmetros medidos durante a produção de açúcar, com ênfase na dificuldade de remoção, ou seja, clarificação do caldo, que se propaga no preço final do açúcar branco ou cristal (Nguyen; Zhang; Doherty, 2015). De acordo com Rein (2007), os principais compostos responsáveis pela alta cor do caldo de cana são pigmentos vegetais (clorofilas, xantofilas e antocianinas), produtos de reação de Maillard, caramelização e degradação da frutose alcalina.

Durante a etapa de refino é realizada a dissolução dos açúcares, que consiste na adição de água até que se obtenha uma calda de concentração brix determinada para produção de açúcar demerara, VHP (Very High Polarization) ou VVHP (Very, Very High Polarization). A clarificação convencional do caldo utiliza polieletrólitos; (como a poliacrilamida que apresentam alto custo); e cal, sem garantia de especificar a cor nos padrões de qualidade VVHP (Prati; Moretti, 2010).

A cor ICUMSA (International Commision for Uniform Methods of Sugar Analysis) é o método mais utilizado para avaliação de cor nas indústrias. A unidade ICUMSA é diretamente proporcional a cor, portanto, quanto mais claro for o açúcar, menor será a unidade (U.I.) (Corrêa, 2016). Esse parâmetro é medido usando, além de medidas de absorbância, a concentração de sacarose à temperatura de $20^{\circ} \mathrm{C}$.

O método mais empregado para clarificação do caldo no Brasil é a sulfitação, em que o enxofre é queimado nos fornos para obtenção do dióxido de enxofre $\left(\mathrm{SO}_{2}\right)$. Portanto, o $\mathrm{SO}_{2}$ gasoso é adicionado ao caldo misto à uma temperatura de $60^{\circ} \mathrm{C}$, atingindo uma faixa de $\mathrm{pH}$ entre 3,8 e 4,2 para seguida alcalinização com cal até um $\mathrm{pH}$ neutro. Nesse processo é utilizada uma proporção de 150 a $300 \mathrm{~g}$ de enxofre por tonelada de açúcar (Mandro, 2016).

O processo de sulfitação tem uso restrito no mercado mundial, e geralmente levam a obtenção do açúcar branco, também fora da especificação do açúcar VVHP. Além disso, estudos indicam que tal processo pode causar problemas respiratórios, como asma e broncoespasmos induzidos. Pessoas com sensibilidade ao sulfito também podem apresentar desconforto na garganta, congestionamento no peito, hipotensão e dermatite de contato (Araújo, 2007; Sartori et al., 2015). O Canadá considera o sulfito como um dos dez alimentos alergênicos prioritários e já dispõe de dura regulamentação nos rótulos de produtos que contém tal composto (Food Allergy Canada, 2020).
Dentro desse contexto, muitos estudos estão sendo desenvolvidos para aplicação de novas tecnologias de remoção de cor, visando processos mais seguros e a diminuição de resíduos tóxicos provenientes de processos de fabricação. Os processos oxidativos avançados (POA) são uma opção alternativa para clarificação, e agem através da oxidação de constituintes orgânicos complexos dificilmente biodegradáveis (Flores, 2008).

Os POAs caracterizam-se pela alta eficiência com poluentes persistentes e efluentes de elevada carga orgânica, ocorrendo através da oxidação de compostos complexos a moléculas simples como $\mathrm{H}_{2} \mathrm{O}, \mathrm{CO}_{2}$. A reação decorre da ação de radicais extremamente oxidantes e não seletivos, como o hidroxil e pode levar até a mineralização dos compostos. Os POAs podem ser classificados em fotoquímicos, que ocorrem sob a incidência de luz, e não fotoquímicos, que ocorrem sob a ausência de luz. Também podem ser chamados de homogêneos com radiação $\left(\mathrm{O}_{3}, / \mathrm{H}_{2} \mathrm{O}_{2} / \mathrm{UV} ; \mathrm{O}_{3} / \mathrm{UV} ;\right.$ FeII/ $\left.\mathrm{H}_{2} \mathrm{O}_{2} / \mathrm{UV}\right)$ sem irradiação $\left(\mathrm{O}_{3} / \mathrm{H}_{2} \mathrm{O}_{2} ; \mathrm{FeII} / \mathrm{H}_{2} \mathrm{O}_{2}\right)$ e heterogêneo com irradiação (semicondutor/UV; semicondutor/ $\mathrm{H}_{2} \mathrm{O}_{2} / \mathrm{UV}$ ) (Benevides et al., 2015; Moreira et al., 2017).

De acordo com Sartori, Magri e Aguiar (2015), a reação de peroxidação é um dos POAs que tem ganhado destaque na etapa de clarificação do caldo, pois sua utilização não necessita de grandes modificações de layout em plantas sucroalcooleiras, além de que o $\mathrm{H}_{2} \mathrm{O}_{2}$ é certificado como como uma substância GRAS (Generally Recognized As Safe) de acordo com Food and Drug Administration (FDA), para o diminuição de cor em alimentos. Para o processo com peróxido de hidrogênio é possível verificar diminuição de parâmetros cruciais como teor de aminoácidos, açúcares redutores, amido, polifenóis, teores de cinzas e viscosidade. A pureza do xarope também é beneficiada com a remoção de não-açúcares. Como os POAs normalmente fornecem a degradação dessas substâncias cromóforas, o açúcar é conservado por longos períodos de armazenamento sem sofrer modificação em sua estrutura. Nesse processo, variáveis como temperatura e $\mathrm{pH}$ devem ser rigorosamente controladas, a fim de se obter um caldo clarificado mantendo o teor de sacarose para posterior fabricação do açúcar (Mandro, 2016). Na clarificação com $\mathrm{H}_{2} \mathrm{O}_{2}$ a faixa de $\mathrm{pH}$ e temperatura de processo é 3 à 7 e 40 à $70^{\circ} \mathrm{C}$ respectivamente (Morilla, 2015).

Segundo Birch (1976), o teor de sacarose presente no caldo de cana-de-açúcar é um dos principais fatores de preocupação na etapa de clarificação, pois como a sacarose é um composto instável, sendo um açúcar não redutor, ela pode se decompor em outros dois açúcares redutores, a glicose e a frutose segundo a reação disposta na Figura 1. 


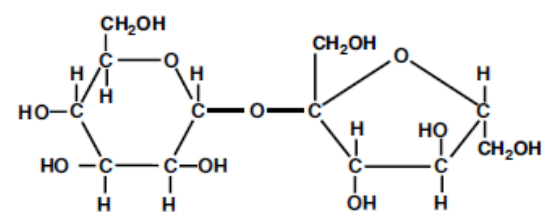

Sacarose

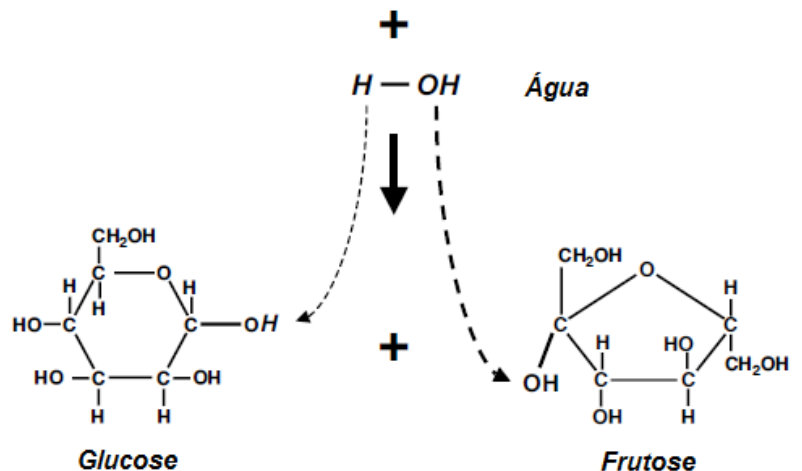

\section{Figura 1 - Reação de inversão da sacarose em glicose e frutose (SHACHMAN, 2005).}

Os açúcares redutores são indesejáveis para a produção de açúcar, pois sua decomposição é direcionada para compostos altamente coloridos, complexos de condensação coloidal, substâncias melassigênicas, como o ácido aspártico, além de que a morfologia dos cristais de sacarose também é alterada com a reação de inversão (Podadera, 2007; Hamerski et al., 2010). Os elementos mais significantes para a inversão da sacarose são temperatura, tempo de residência da solução no processo e $\mathrm{pH}$. Quando se trabalha com meios ácidos a inversão pode ser ainda mais estimulada à elevadas temperaturas. Para um meio básico e nas mesmas condições de temperatura, ao invés da formação de glicose e frutose, pode haver a hidrólise da sacarose, impulsionando a formação de outros compostos, como a acetona, gliceraldeídos, ácido lático, ácido acético, ácido fórmico, dióxido de carbono, entre outros (Silva; Assunção; Sousa Filho, 2017).

Este trabalho tem como objetivo avaliar o método de clarificação do caldo de cana-de-açúcar através da reação de peroxidação utilizando o efeito fotocatalítico com e sem a incidência da luz ultravioleta para redução da cor ICUMSA, parâmetro que é usado para classificação comercial do açúcar no Brasil. Variáveis como $\mathrm{pH}$ e brix também foram analisados afim de se obter um caldo clarificado e de maior valor agregado.

\section{MATERIAIS E MÉTODOS}

Para o processo de clarificação do caldo da cana-deaçúcar utilizou-se processo oxidativo avançado através da reação de peroxidação, com e sem o efeito da luz ultravioleta, adaptado de Mandro (2016).

Os experimentos de peroxidação foram realizados em uma unidade experimental completamente fechada para que não houvesse interferência de luz externa. $\mathrm{O}$ desenho esquemático da unidade utilizada para o processo fotocatalítico está disposto na Figura 2.

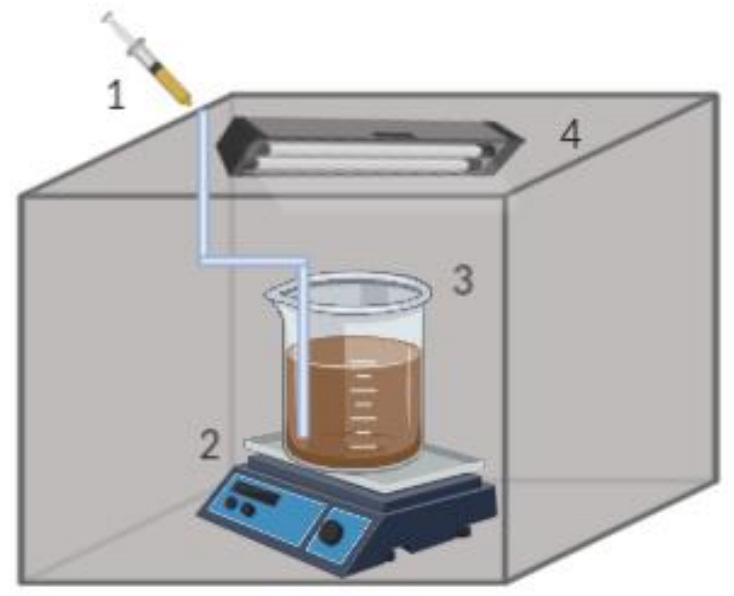

Figura 2 - Esquema ilustrativo da unidade fotocatalítica.

Sendo,

(1) Amostragem;

(2) Agitador magnético;

(3) Béquer com $50 \mathrm{~mL}$ de caldo filtrado;

(4) Lâmpada UV.

As alíquotas eram retiradas com o auxílio de uma mangueira ligada a uma seringa na área externa da unidade, isso permitiu que as amostras pudessem ser coletadas sem interrupção da reação. $\mathrm{O}$ procedimento experimental do processo de clarificação ocorreu de acordo com o fluxograma disposto na Figura 3.

Amostras do caldo não filtrado foram coletadas para medidas de absorbância e brix. Posteriormente, o caldo foi filtrado com o auxílio de um papel filtro e mediu-se o $\mathrm{pH}$ inicial. A duração dos experimentos foi de $1 \mathrm{~h}$, com coletas para os tempos de: 5, 10, 15, 20, 30, 45 e 60 minutos. Nos estudos de clarificação foram consideradas três diferentes situações:

\subsection{Reação do caldo com peróxido de hidrogênio sem a incidência de luz UV}

Transferiu-se $50 \mathrm{~mL}$ de caldo filtrado para um béquer de $600 \mathrm{~mL}$ mantendo sob a agitação. Foi adicionado peróxido de hidrogênio ao caldo em duas diferentes concentrações $(0,5$ $\mathrm{mol} / \mathrm{L} \mathrm{e} 1,0 \mathrm{~mol} / \mathrm{L})$, e iniciou-se a contagem do tempo, coletando alíquotas de $0,5 \mathrm{~mL}$ do caldo clarificado em diferentes tempos para análise da absorbância. Após 1 hora de reação, retirou-se a alíquota final e mediu-se o $\mathrm{pH}$ e o brix.

\subsection{Reação do caldo sem peróxido de hidrogênio sob a incidência de luz UV}

Da mesma maneira $50 \mathrm{~mL}$ de caldo filtrado foram transferidos para um béquer de $600 \mathrm{~mL}$ e mantido sob a agitação dentro de uma câmara totalmente fechada com a incidência de luz ultravioleta. A fonte de luz foi uma lâmpada de vapor mercúrio de $80 \mathrm{~W}$, sem o bulbo de vidro. Alíquotas de $0,5 \mathrm{~mL}$ da solução foram retiradas com o auxílio de uma mangueira e uma seringa durante o período de 1 hora. Ao fim da corrida, mediu-se o $\mathrm{pH}$ e o brix. 


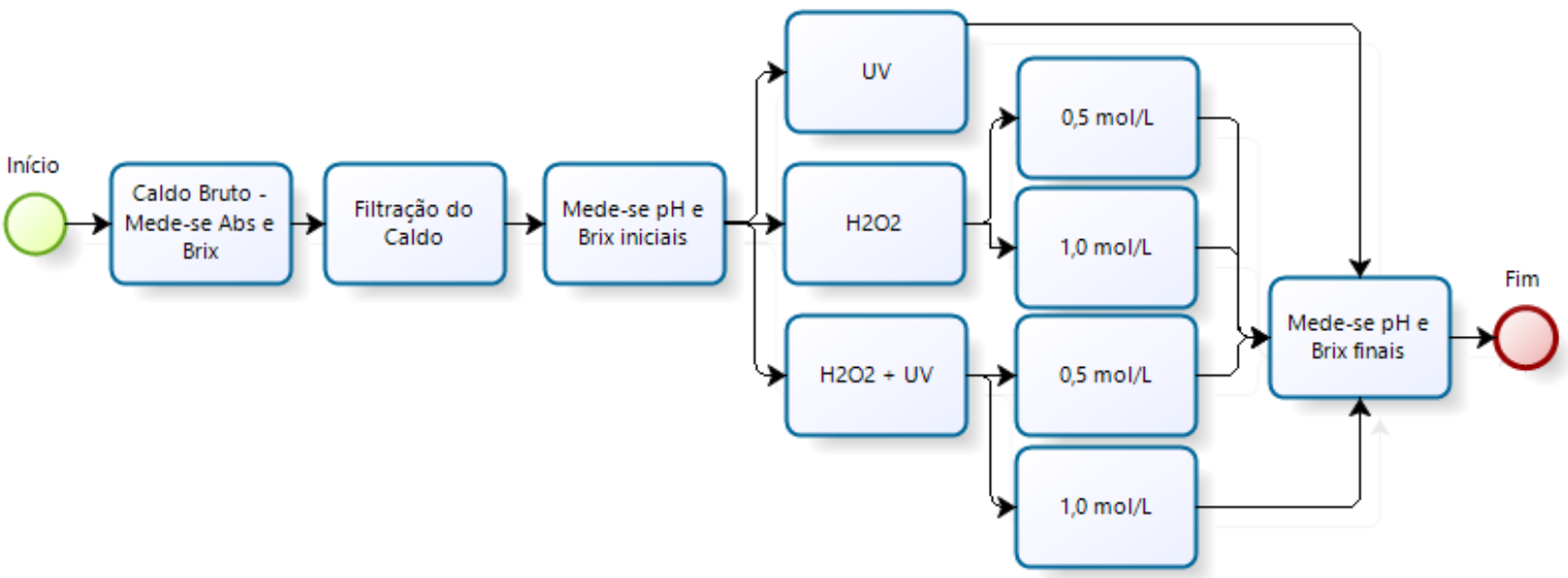

Figura 3 - Fluxograma representativo do processo de clarificação.

\subsection{Reação do caldo com peróxido de hidrogênio com a} incidência de luz UV

Igualmente, $50 \mathrm{~mL}$ de caldo filtrado foram transferidos para um béquer de $600 \mathrm{~mL}$ e mantido sob a agitação. Foi adicionado peróxido de hidrogênio ao caldo em duas diferentes concentrações $(0,5 \mathrm{~mol} / \mathrm{L}$ e $1,0 \mathrm{~mol} / \mathrm{L})$. Todo o aparato, béquer e agitador foram colocados dentro de uma câmara totalmente fechada com a incidência de luz ultravioleta, através de uma lâmpada de mercúrio, $80 \mathrm{~W}$, sem bulbo. Alíquotas de $0,5 \mathrm{~mL}$ da solução foram coletadas durante 1 hora. Ao fim da corrida, mediu-se o $\mathrm{pH}$ e o brix.

Tabela 1.

Os dados dos três experimentos estão apresentados na

Tabela 1 - Medidas de $\mathrm{pH}$ e brix para as corridas que avaliam respectivamente: a presença do $\mathrm{H}_{2} \mathrm{O}_{2}$ sob a incidência de luz $U V$, apenas a ação do $\mathrm{H}_{2} \mathrm{O}_{2}$ e por fim os resultados da luz $\mathrm{UV}$.

\begin{tabular}{cccccc}
\hline & $\mathbf{U V}+\mathbf{H}_{2} \mathbf{O}_{2}$ & \multicolumn{3}{c}{$\mathbf{H}_{2} \mathbf{O}_{2}$} & $\mathbf{U V}$ \\
$\begin{array}{c}\text { Concentração de } \\
\text { H2 }\end{array}$ & $\mathbf{0 , 5}$ & $\mathbf{1 , 0}$ & $\mathbf{0 , 5}$ & $\mathbf{1 , 0}$ & - \\
\hline $\mathbf{p H}$ (inol/L) & & & & & \\
pH (final) & 5,08 & 5,08 & 5,08 & 5,08 & 5,08 \\
Brix (inicial) & 4,73 & 4,11 & 5,04 & 4,51 & 4,36 \\
Brix (final) & 21,0 & 21,0 & 21,0 & 21,0 & 21,0 \\
\hline
\end{tabular}

Todas as análises de absorbância foram realizadas em espectrofotômetro com o comprimento de onda de $420 \mathrm{~nm}$, utilizando cubeta de quartzo com $1 \mathrm{~cm}$ de comprimento interno. Já para o brix, foi utilizado um refratômetro digital, sendo necessária uma pequena quantidade de amostra, cerca de 2 gotas. Mediu-se o pH no início e no fim de cada corrida, utilizando um pHmetro de bancada do tipo pH 250, sempre aguardando os valores se estabilizarem.

A cor ICUMSA foi determinada através da Equação 1 (Umebara, 2010).

$$
=\frac{-}{*} * 1000
$$

Sendo,

Abs: absorbância da alíquota à $420 \mathrm{~nm}$;

b: comprimento interno da cubeta;

c: concentração de sacarose $(\mathrm{g} / \mathrm{mL})$.

A concentração de sacarose foi aproximada considerando uma solução de $20^{\circ}$ brix contendo $0,180 \mathrm{~g} / \mathrm{mL}$ de sacarose, contendo $90 \%$ de pureza (Basso et al., 2011).

\section{RESULTADOS E DISCUSSÃO}

Os dados de absorbância obtidos de acordo com os três experimentos avaliados estão dispostos na Tabela 2.

Tabela 2 - Dados de absorbância do caldo durante o processo de clarificação.

\begin{tabular}{cccccc}
\hline $\begin{array}{c}\text { Tempo } \\
(\mathbf{m i n})\end{array}$ & $\begin{array}{c}\mathbf{0 , 5} \\
(\mathbf{m o l} / \mathbf{L})\end{array}$ & $\begin{array}{c}\mathbf{1 , 0} \\
(\mathbf{m o l} / \mathbf{L})\end{array}$ & $\begin{array}{c}\mathbf{0 , 5} \\
(\mathbf{m o l} / \mathbf{L})\end{array}$ & $\begin{array}{c}\mathbf{1 , 0} \\
(\mathbf{m o l} / \mathbf{L})\end{array}$ & - \\
\hline $\mathbf{0}$ & 0,902 & 0,902 & 0,902 & 0,902 & 0,902 \\
$\mathbf{5}$ & 0,968 & 0,736 & 0,631 & 0,727 & 1,046 \\
$\mathbf{1 0}$ & 0,852 & 0,814 & 0,722 & 0,720 & 1,216 \\
$\mathbf{1 5}$ & 0,633 & 0,824 & 0,756 & 0,818 & 1,237 \\
$\mathbf{2 0}$ & 0,710 & 0,821 & 0,627 & 0,793 & 1,130 \\
$\mathbf{3 0}$ & 0,514 & 0,868 & 0,691 & 0,761 & 1,166 \\
$\mathbf{4 5}$ & 0,480 & 0,751 & 0,680 & 0,797 & 1,189 \\
$\mathbf{6 0}$ & 0,370 & 0,606 & 0,628 & 0,784 & 1,043 \\
\hline
\end{tabular}

A partir dos resultados de absorbância, foi possível calcular os parâmetros de cor ICUMSA, e assim, realizar um comparativo do percentual de redução de cor disposto no gráfico da Figura 4 para os três processos que ocorreram sob a incidência de luz UV. 


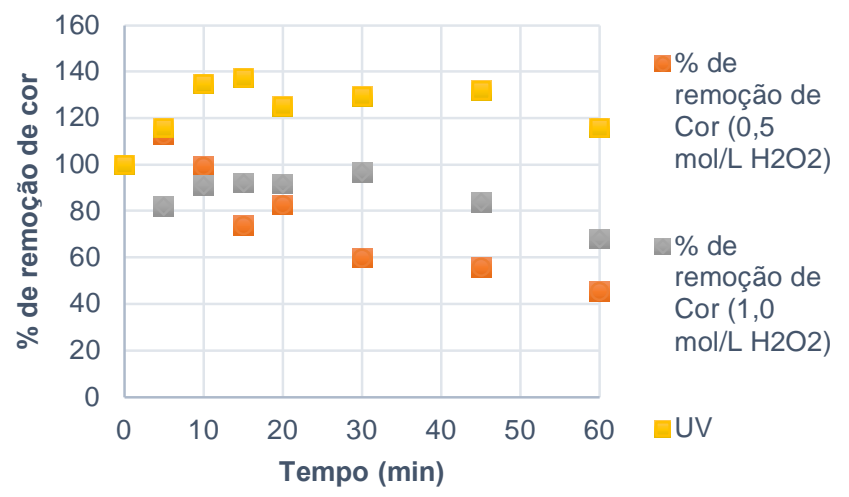

Figura 4 - Curvas de percentual de remoção de cor para o processo de clarificação utilizando peróxido de hidrogênio sob o efeito da luz ultravioleta.

Através do gráfico, pode-se observar três curvas distintas, onde há uma maior eficiência para o processo com a menor concentração de peróxido de hidrogênio somada a ação da luz UV, havendo uma queda na coloração do caldo original de aproximadamente $55 \%$. Ao verificar o brix, pode-se perceber o decréscimo de 21,0 para $18,9^{\circ}$ brix mediante $\mathrm{pH}$ de 4,73 . Para essa situação, percebe-se que o incremento de luz acelera o processo de clarificação, agindo como um catalisador para a reação de peroxidação.

Dobrando a concentração de peróxido, obtém-se uma menor eficiência do processo, sendo a redução da cor ICUMSA de apenas $32 \%$. Tal fato é decorrente de reações paralelas que ocorrem na degradação do substrato $(\mathrm{S})$, onde os radicais $\mathrm{HO}^{\circ}$ tem a possibilidade de reagir diretamente com o $\mathrm{S}$, levando à oxidação, como na Equação (2). Quando em excesso de $\mathrm{H}_{2} \mathrm{O}_{2}$ e $\mathrm{HO}^{\circ}$, o radical hidroxila do meio é abstraído, representado pela Equação 2, o que leva a formação de hidroperoxil $\left(\mathrm{HO}_{2}{ }^{\circ}\right)$, que se caracteriza por um composto com potencial de oxidação menor. Subsequentemente, o radical hidroperoxil pode reagir com o $\mathrm{H}_{2} \mathrm{O}_{2}$, formando radicais hidroxila que levam à formação de ainda mais $\mathrm{H}_{2} \mathrm{O}_{2}$ de acordo com as Equações (4 e 5). O que forma uma tendência em cadeia para reduzir a eficiência do processo oxidativo avançado (Andreozzi et al., 1999; Dantas, 2005).

$H O^{o}+S \rightarrow S^{o}$

$\mathrm{H}_{2} \mathrm{O}_{2}+\mathrm{HO}^{o} \rightarrow \mathrm{HO}_{2}{ }^{o}+\mathrm{H}_{2} \mathrm{O}$

$\mathrm{H}_{2} \mathrm{O}_{2}+\mathrm{HO}_{2}{ }^{o} \rightarrow \mathrm{HO}^{\circ}+\mathrm{O}_{2}+\mathrm{H}_{2} \mathrm{O}$

$2 \mathrm{HO}^{\circ} \rightarrow \mathrm{H}_{2} \mathrm{O}_{2}+\mathrm{O}_{2}$

Para o procedimento experimental em que o caldo ficou sob a incidência de luz UV, ou seja, sem a ação do clarificante $\left(\mathrm{H}_{2} \mathrm{O}_{2}\right)$, não se observou a diminuição da cor, indicando que apenas o efeito da radiação não tem nenhum impacto sobre a clarificação.

Os resultados dos processos que utilizaram somente a reação com peróxido de hidrogênio $(0,5$ e $1,0 \mathrm{~mol} / \mathrm{L})$, sem o efeito da luz ultravioleta, estão mostrados no gráfico da Figura 5.

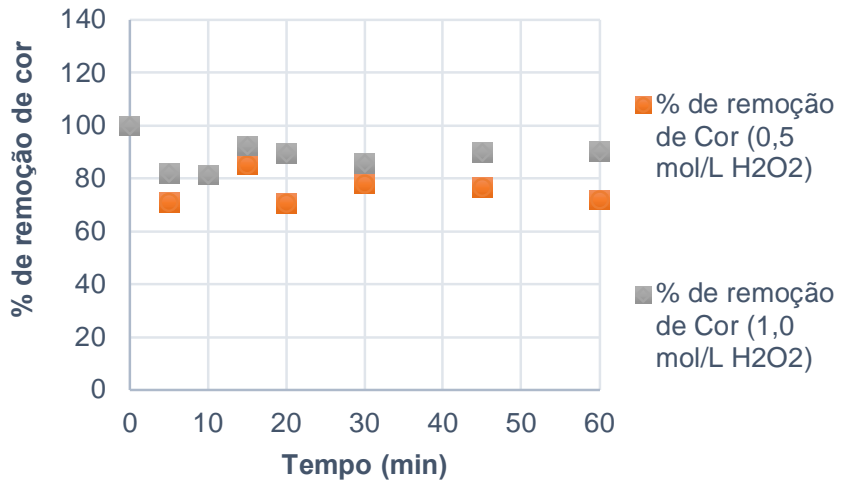

Figura 5 - Curvas de percentual de remoção de cor para o processo de clarificação utilizando peróxido de hidrogênio sem o efeito da luz ultravioleta.

De acordo com o gráfico, nota-se que apenas a ação do peróxido não traz resultados tão eficientes quando comparado ao processo com a luz UV, com uma remoção de cor de $28 \%$ e $10 \%$, para as corridas com $0,5 \mathrm{~mol} / \mathrm{L}$ e $1,0 \mathrm{~mol} / \mathrm{L}$ de $\mathrm{H}_{2} \mathrm{O}_{2}$, respectivamente.

Um fator que deve ser analisado é a degradação da sacarose, que não é um composto totalmente estável, se decompondo em glicose e frutose. No caso em questão, há três variáveis que influenciam fortemente a degradação da sacarose, que são: temperatura, $\mathrm{pH}$ e concentração de $\mathrm{H}_{2} \mathrm{O}_{2}$. Trabalhos como o de Panpae, et al. (2008) que estudaram a degradação da sacarose através da influência do pH e temperatura, chegaram à conclusão de que ao submeter a sacarose a altas temperaturas, o teor de açúcares redutores aumentavam enquanto a sacarose era invertida, portanto, a temperatura é um dos parâmetros que aceleram o processo de inversão.

Segundo Zurita (2008), a taxa de degradação da sacarose também pode ser estimulada pela concentração dos reagentes e uso de catalisadores. Alguns dos processos que podem levar a transformação dos açúcares são a incidência de luz UV, raio X, nêutrons e micro-ondas.

De acordo com Claude e Ubbink (2006), para soluções com pH mais ácidos ou acima de 8,0, existem chances significativas de degradação, sendo uma faixa de $\mathrm{pH}$ ideal próxima de 5,5 .

Avaliando o processo em que houve maior clarificação, ou seja, na condição de $0,5 \mathrm{~mol} / \mathrm{L} \mathrm{H}_{2} \mathrm{O}_{2}+\mathrm{UV}$, não houve queda considerável do $\mathrm{pH}$ final $(4,73)$ com relação ao inicial $(5,08)$. Para a medida de brix, apenas 1,1 ${ }^{\circ}$ brix de decréscimo foi acometido ao caldo. Isso representa que o processo de clarificação implementado não degradou significativamente a sacarose, o que é sustentando pela baixa variação no $\mathrm{pH}$.

Segundo Sartori (2014), que verificou o percentual de remoção da cor ICUMSA para o caldo de cana-de-açúcar, concentrações abaixo de $0,017 \mathrm{~mol} / \mathrm{L}$ de peróxido de hidrogênio não são suficientes para perceber a degradação de substâncias cromóforas. No entanto, a temperatura foi mantida entre $40 \mathrm{e}$ $70^{\circ} \mathrm{C}$, obtendo condições ótimas para um $\mathrm{pH}$ abaixo de 7,0, utilizando qualquer concentração de peróxido até $0,035 \mathrm{~mol} / \mathrm{L}$. Verifica-se então que a peroxidação realizada sob a incidência de luz ultravioleta não modifica o pH de forma significativa, e não se faz necessária a sua correção, como há no processo de 
sulfitação comumente empregado em indústrias sucroalcooleiras, tornando o processo ainda mais viável economicamente.

\section{CONCLUSÃO}

Portanto, é possível afirmar que o processo mais eficiente para a clarificação do caldo foi com a adição de 0,5 mol/L de $\mathrm{H}_{2} \mathrm{O}_{2}$ sob a incidência de luz UV, não havendo decaimento significativo de $\mathrm{pH}$ e brix o que levaria à inversão da sacarose. Aumentando a concentração de peróxido, há uma queda na eficiência devido a ação de reações paralelas decorrentes do excesso de reagente $\left(\mathrm{H}_{2} \mathrm{O}_{2}\right)$. Outro ponto importante foi que sob a ausência de luz UV, há uma clarificação insignificante quando comparada a reação de peroxidação somada a incidência de luz UV.

Melhores resultados de clarificação poderiam ser obtidos encontrando uma concentração ótima de $\mathrm{H}_{2} \mathrm{O}_{2}$, em que o custo com esse reagente fosse minimizado. Para experimentos futuros, o tempo de reação pode ser aprimorado, verificando se o decaimento de cor seria mais elevado com o prolongamento da reação. A potência da lâmpada somada a temperatura do meio também são condições de operação que devem ser avaliadas para potencializar a clarificação.

\section{REFERÊNCIAS}

ANDREOZZI, R.; CAPRIO, V.; INSOLA, A.; MAROTTA, R., Advanced Oxidation Process (AOP) for water purification and recovery. Catalysis Today, Amsterdam, v. 53, n. 1, p. 51-59, out. 1999.

ARAÚJO, F.A.D.; Processo de clarificação do caldo de cana pelo método da bicarbonatação. Revista Ciências e Tecnologia. Ano 1, n. 1, p. 1-5, 2007.

BASSO, L.; BASSO, T.; ROCHA, S. Ethanol production in Brazil: the industrial process and its impact on yeast fermentation. Biofuel production - Recent Developments and Prospects, v. 1530, p. 85ї 100, 2011.

BENEVIDES, J. A. J.; MARINHO, G. M. Degradação de pesticidas por fungos ï uma revisão. HOLOS, Ceará, Ano 31, Vol. 2, 2015.

BIRCH, G. G. Structural relationships of sugar to taste. Critical Reviews in Food Science and Nutrition, v. 8, n.1, p. 57 95, 1976.

CLAUDE, J.; UBBINK, J. Thermal degradation of carbohydrate polymers in amorphous states: a physical study including colorimetry. Food Chemistry, Oxford, v. 96, n. 3, p. 402-410, 2006.

CORRÊA, N. T. Cinética de redução de cor ICUMSA em caldo de cana-de-açúcar por ozonização em reator RPA/CSTR para produção de sulfur-free de açúcar branco. 2016. 100 f. Dissertação (Mestrado em Cicências de Microbiologia Agrícola) ï Universidade de São Paulo, SP, 2016.

DANTAS, T. L. P. Decomposição de peróxido de hidrogênio em um catalisador híbrido e oxidação avançada de efluente têxtil por reagente fenton modificado. 2005. 106 f. Dissertação (Mestrado em Engenharia Química) ï Universidade Federal de Santa Catarina, SC, 2005.

FLORES, R. G. Aplicação de processos oxidativos avançados homogêneos e heterogêneos na degradação de efluentes de curtume. 2008. 76 f. Dissertação (Mestrado em Engenharia Civil) ï Universidade Federal de Santa Maria, RS, 2008.

FOOD ALLERGY CANADA. What is food allergy? Sulphites. 2020. Disponível em: <https://foodallergycanada.ca/food-allergy-basics/foodallergies-101/what-are-food-allergies/sulphites/>. Acessado em 20 jun., 2020.

HAMERSKI, F.; AQUINO, A. D.; NDIAYE, P. M. Clarificação do cado de cana-de-açúcar por carbonatação ï ensaios preliminaries. Acta Scientiarum. Technology, Curitiba, p. 337-341. 22 abr. 2010.

MANDRO, J. L. Processo de peroxidação do açúcar tipo VHP na produção de açúcar refinado: implicações químicas, tecnológicas e microbiológicas. 2016. 96 f. Dissertação (Mestrado em Ciências de Microbiologia Agrícola) $\ddot{i}$ Universidade de São Paulo, SP, 2016.

MOREIRA, A. J.; BORGES, A. C.; GOUVEIA, L. F. C.; MACLEOD, T. C. O.; FRESCHI, G. P. G.. The process of atrazine degradation, its mechanism, and formation of metabolites using UV and UV/MV photolysis. Journal of Photochemistry and Photobiology A: Chemistry, [s.1.], v. 347, p. 160-167, out. 2017.

MORILLA, C. H. G. Validade econômico-financeira da substituição do dióxido de enxofre pelo peróxido de hidrogênio na cadeia produtiva do açúcar. 2015. 105 f. Dissertação (Mestrado em Ciências de Tecnologia dos Alimentos) ï Universidade de São Paulo, SP, 2015.

NGUYEN, Danny M. T.; ZHANG, Zhanying; DOHERTY, William O. S.. Degradation of Hydroxycinnamic Acid Mixtures in Aqueous Sucrose Solutions by the Fenton Process. Journal Of Agricultural And Food Chemistry, [s.1.], v. 63, n. 5, p. 1582-1592, 29 jan. 2015.

OGANDO, Felipe Iwagaki Braga; AGUIAR, Claudio Lima de; VIOTTO, João Vitor Napolitano; HEREDIA, Francisco José; HERNANZ, Dolores. Removal of phenolic, turbidity and color in sugarcane juice by electrocoagulation as a sulfur-free process. Food Research International, [s.1.], v. 122, p. 643-652, ago. 2019.

PANPAE, K.; JATURONRUSMEE, W.; MINGVANISH, W.; NUNTIWATTANAWONG, C.; CHUNWISET, S.; SANTUDROB, K.; TRIPHANPITAK, S. Minimization of sucrose losses in sugar industry by $\mathrm{pH}$ and temperature optimization. The Malaysian Journal of Analytical Sciences, Bangi, v. 12, n. 3, p. 513-519, 2008.

PODADERA, P. Estudo das propriedades do açúcar líquido invertido processado com radiação gama e feixe de elétrons. 2007. 99 f. Tese (Doutorado em Ciências de Tecnologia Nuclear) ï Instituto de Pesquisas Energéticas e Nucleares, Universidade de São Paulo, SP, 2007.

PRATI, P.; MORETTI, R. H. Study of clarification process of sugar cane juice for consumption. Ciência e Tecnologia dos Alimentos, v. 30, n. 3, 2010.

REIN, P. W. Cane sugar engineering. Berlin: Bartens $17 ̈ 768$. 2007.

SARTORI, J. A. S. Cinética Química do decaimento de cor ICUMSA de caldo de cana-de-açúcar por reação de oxidação com peróxido de hidrogênio em reatores de fase homogênea. 2014. 116 f. Dissertação (Mestrado em Cicência e Tecnologia dos Alimentos) ï Escola Superior de Agricultura Luiz de Queiroz, Universidade de São 
Paulo, SP, 2014.

SARTORI, J. A. S; MAGRI, N. T. C; AGUIAR, C. L. Clarificação do caldo da cana-de-açúcar por peróxido de hidrogênio: efeito da presença de dextrana. Brazilian Journal of Food Tecnology. Campinas, v. 18, n. 4, p. 299-306, 2015.

SACHMAN, M. The Soft Drinks Companion - A Technical Handbook for the Beverage Industry. Boca Raton, EUA: CRC Press, 2005.

SILVA, C. C.; ASSUNÇÃO, L. F.; SOUSA FILHO, J. W. Determinação da inversão da sacarose em caldo de canade-açúcarsubmetido a tratamento por irradiação microondas para produção de açúcar. Revista Brasileira de
Ciência, Tecnologia e Inovação (RBCTI), v. 2, n.1, p. 6-12 jan./jun, 2017.

UMEBARA, T. Microfiltração de caldo de cana: caracterização do caldo permeado e retentado. 2010. 100 f. Dissertação (Mestrado em Tecnologia de Alimentos) ï Universidade Federal do Paraná, PR, 2010.

ZURITA, E. J. C. Avaliação da Inversão da Sacarose em um Sistema de Evaporação (Evaporador de Filme Descendente com Promotor de Película). 2008. 95 f. Tese (Doutorado em Engenharia Química) ï Universidade de São Paulo, SP, 2008. 